Result(s)* Among 33 tumors, 18 were serous, 12 mucinous and 3 sero-mucinous. Molecular analysis of tumors allowed us to detect mutations in genes involved in the MAP Kinase (MAPK) cascade and in the DNA repair mechanism. Our initial analysis revealed an association between defects in DNA Double-Strand Break repair and occurrence of mucinous BOT, in $75 \%$ of cases. Mutations affecting MAPK signaling pathway were detected in $46.9 \%$ of BOT.

Conclusion* Here we report the molecular profile of BOT in the Lebanese population. This is the first study associating the DNA repair pathway to BOT. The inclusion of further patients is essential to validate our hypothesis and to better delineate the mechanisms of the disease, thus allowing the implementation of targeted therapeutic approaches.

\section{NEOADJUVANT CHEMOTHERAPY AND FERTILITY SPARING SURGERY IN ADVANCED MALIGNANT GERM CELL TUMOR OVARY}

${ }^{1} \mathrm{~A}$ JS*, 'S Sambasivan, ${ }^{1} \mathrm{PN}$ Rema, ${ }^{2} \mathrm{~S}$ Ranjith J, ${ }^{3} \mathrm{FV}$ James, ${ }^{4} \mathrm{~J}$ Krishna. ${ }^{1}$ Regional Cancer Centre, Thiruvananthapuram, Gynecological oncology, Thiruvananthapuram, India; ${ }^{2}$ Regional Cancer Centre, Thiruvananthapuram, Surgical oncology, Thiruvananthapuram, India; ${ }^{3}$ Regional Cancer Centre, Thiruvananthapuram, Radiation Oncology, Thiruvananthapuram, India; ${ }^{4}$ Regional Cancer Centre, Thiruvananthapuram, Cancer Epidemiology and Biostatistics, Thiruvananthapuram, India

\subsection{6/ijgc-2021-ESGO.440}

Introduction/Background* Malignant Germ cell tumours (MGCTs) are rare tumours that account for 2\% - 3\% of all ovarian cancers. Being highly chemosensitive, fertility sparing surgery whenever feasible with or without adjuvant chemotherapy, is the standard treatment approach in these patients. Upfront fertility sparing surgery may not be feasible in all patients with advanced stage disease due to poor performance status, bilateral ovarian disease or large tumours infiltrating the uterus. Neoadjuvant chemotherapy (NACT) followed by conservative surgery might be considered for such patients. This study aimed to analyse the outcome of patients with advanced malignant germ cell tumour ovary who underwent NACT followed by surgery and to assess the menstrual and reproductive function in those patients who underwent conservative surgery.

Methodology A retrospective study of 28 patients who underwent debulking surgery following neoadjuvant chemotherapy for advanced malignant germ cell tumour ovary from January 2008 to March 2019. Clinical information and follow up data till December 2020 were collected from medical records.

Result(s)* The median follow up period was 76 months (range 7 to 133 months). The median age was 16.5 years (range 7 to 31years). Twenty four $(85.8 \%)$ patients underwent fertility sparing surgery. Complete pathological response was seen in $25(89.3 \%)$ patients. Two patients were lost to follow up, one of whom was pregnant at the time of the last follow up. One patient, who initially presented with stage IV Dysgerminoma and underwent bilateral salpingo- oophorectomy following chemotherapy, died 6 months after surgery due to disease recurrence. Of the remaining patients, 17 reported menstruation following treatment. Two patients were diagnosed with primary amenorrhoea and 2 were still premenarchal. Three patients tried for pregnancy and had a total of 4 pregnancies. Conclusion* NACT followed by interval debulking surgery might be considered in patients with advanced malignant germ cell tumour. In patients with poor performance status and high tumor load, neoadjuvant chemotherapy makes fertility sparing surgery feasible with a good reproductive outcome.

\section{NOGGO OV-42/MAMOC: RUCAPARIB MAINTENANCE AFTER BEVACIZUMAB MAINTENANCE FOLLOWING CARBOPLATIN BASED FIRST LINE CHEMOTHERAPY IN OVCA PATIENTS}

${ }^{1} \mathrm{E}$ Braicu* ${ }^{2} \mathrm{P}$ Wimberger, ${ }^{3,4} \mathrm{~A}$ Relógio, ${ }^{5} \mathrm{~J}$ Dysarz, ${ }^{6} \mathrm{~J}$ Gerber, ${ }^{7} \mathrm{M}$ Eichbaum, ${ }^{8} \mathrm{M}$ Derya ${ }^{1} \mathrm{R}$ Chekerov, ${ }^{1 \mathrm{~J}}$ Grabowksi, ${ }^{9} \mathrm{M}$ Rose, ${ }^{10} \mathrm{M}$ Endres, ${ }^{1} \mathrm{C}$ Scheibenbogen, ${ }^{1} \mathrm{H}$ Woopen, 1J Sehouli. 'Charité Universitätsmedizin Berlin, Klinik für Gynäkologie, Campus Virchow Klinikum, Berlin, Germany; ${ }^{2}$ Universitätsklinikum Carl Gustav Carus, Klinik und Poliklinik für Frauenheilkunde und Geburtshilfe, Dresden, Germany; ${ }^{3}$ Systems Biology of Cancer Molecular Cancer Research Center (MKFZ), Institute for Theoretical Biology (ITB) Charité Universitätsmedizin Berlin, Berlin, Germany; ${ }^{4}$ Department of Human Medicine, Institute for Systems Medicine, MSH Medical School Hamburg, Germany; ${ }^{5}$ NOGGO e.V. NordOstdeutsche Gesellschaft für Gynäkologische Onkologie, Berlin, Germany; ${ }^{6}$ Städtisches Klinikum Dessau, Klinik für Frauenheilkunde und Geburtshilfe, Dessau, Germany; ${ }^{7}$ Helios Dr. Horst Schmidt Kliniken Wiesbaden, Klinik für Frauenheilkunde und Geburtshilfe, Wiesbaden, Germany; ${ }^{8}$ Caritas Klinikum Saarbrücken, Zentrum für Geburtshilfe und Frauenheilkunde, Saarbrücken, Germany; ${ }^{9}$ Charité Universitätsmedizin Berlin, Medizinische Klinik m.S. Psychosomatik, Campus Benjamin Franklin, Berlin, Germany; ${ }^{10}$ Charité Universitätsmedizin Berlin, Klinik für Neurologie mit Experimenteller Neurologie, Berlin, Germany

\subsection{6/ijgc-2021-ESGO.441}

Introduction/Background* Ovarian cancer (OC) is associated with the highest mortality rates among gynecological malignancies, with most patients being diagnosed in advanced stages. The most common histological subtype is high grade serous OC, which is characterized by deficiency in homologous recombination. Debulking surgery, followed by platinum based chemotherapy and bevacizumab (bev), followed by maintenance therapy with bev, is the standard therapy for advanced BRCA wild type (BRCAwt) OC patients in Germany. BRCA mutant patients will receive maintenance with olaparib, according to SOLO1 data. The anticancer effects of PARP inhibitors (PARPi) seem to be increased by the addition of antiangiogenic drugs. Preclinical data showed increased HRD in tumors pretreated with bev, and clinical trials showed a benefit of the combination of antiangiogenic drugs and PARPi vs. PARPi alone. NOGGO Ov-42/MAMOC trial (NCT04227522) is a phase 3, randomized, placebo-controlled study evaluating rucaparib maintenance following bevacizumab maintenance for the treatment of advanced primary high grade BRCAwt OC.

Methodology 190 patients with histologically confirmed advanced (FIGO stage IIIA- IV of the 2014 FIGO classification) high grade serous or high grade endometrioid (based on local histopathological findings) OC, fallopian tube cancer, primary peritoneal cancer or clear cell carcinoma of the ovary will be randomized $2: 1$ to receive either rucaparib $600 \mathrm{mg}$ BID or placebo as maintenance therapy following first line chemotherapy with 6 cycles of Carboplatin/Paclitaxel and at least 12 cycles of bev, given together with chemotherapy and as maintenance. Only BRCAwt patients will be included in the trial. Randomization is stratified by surgery planned timepoint (neoadjuvant vs. adjuvant), surgical outcome (no residual tumor mass vs. residual tumor mass), response to chemotherapy followed by bev (CR/NED vs. PR/SD) and study center. Treatment will continue for 24 months or until disease progression, unacceptable toxicity, or withdrawal. Primary endpoint is PFS in BRCAwt patients per RECIST v1.1. Secondary endpoints are PFS2, quality of life (EORTC QLQ-C30/OV28, Fatigue Symptom Inventory, SF-12, PROC-CTCAE, Everyday 